\title{
MODELO PARA LA EVALUACIÓN DINÁMICA DE LA GESTIÓN DE RESIDUOS ORDINARIOS DE LA CIUDAD DE BOGOTÁ Y SU INFLUENCIA EN EL ÍNDICE DE CALIDAD AMBIENTAL URBANA
}

\author{
MODEL FOR THE DYNAMIC MANAGEMENT ASSESSMENT \\ OF ORDINARY WASTE IN BOGOTA AND ITS INFLUENCE \\ ON THE URBAN ENVIRONMENTAL QUALITY INDEX
}

\author{
1 Jennifer Rodríguez-Andrade \\ 2 Danny Ibarra-Vega \\ ${ }^{1}$ Ingeniera ambiental, integrante del Semillero de Investigación en Ingeniería Ambiental \\ y Bioprocesos, Universidad Sergio Arboleda, Bogotá, Colombia. \\ ${ }^{2}$ PhD., MSC., Gestión Ambiental. Investigador de IRCACS-International Research Center \\ for Applied Complexity Sciences, Bogotá, Colombia. \\ 1 jennifer.rodrigueza@correo.usa.edu.co \\ 2 ingdanny09@hotmail.com
}

\section{RESUMEN}

El objetivo de este artículo fue elaborar un modelo para la evaluación dinámica de la gestión de residuos ordinarios en Bogotá y evaluar su influencia en los indicadores de residuos aprovechados y dispuestos adecuadamente, pertenecientes al Índice de Calidad Ambiental Urbana. Para la construcción del modelo se utilizó la metodología de Dinámica de Sistemas, la cual permitió evaluar distintos escenarios de gestión. Se propusieron cuatro escenarios de comportamiento: el primero representaba el aumento en la separación en la fuente; el segundo, el aumento de aprovechamiento a un $20 \%$; el tercero reunía los dos anteriores; y, finalmente, el cuarto, buscaba incentivar la separación en la fuente y llevar el

aprovechamiento a un $50 \%$. Teniendo en cuenta las simulaciones del modelo, se demuestra que es urgente crear estrategias de educación a la población generadora de residuos, para incentivar la separación en la fuente; además, es importante impulsar el aprovechamiento, para que, de forma conjunta, se impacte la vida útil del relleno sanitario y se mejoren los resultados de los indicadores analizados.

Palabras clave: Gestión integral de residuos, modelo matemático, modelo dinámico.

\section{ABSTRACT}

The objective of this article was to develop a model for the dynamic management 
assessment of the ordinary waste of Bogota and to assess its influence in the indicators of used and properly disposed waste, which are part of the Urban Environmental Quality Index. For the construction of the model, the Systems Dynamic methodology was applied, which allowed the assessment of different management scenarios. Four scenarios were proposed: the first one represented the increase in the separation at the source; the second one showed an increase of $20 \%$ in the recycling; the third one collected the previous two; and, finally, the fourth one looked for encouraging the separation at the source and bring to $50 \%$ the recycling. Considering the model simulations, it is evident that it is urgent to elaborate education strategies for the population to promote the separation at the source; furthermore, it is important to improve the recycling in order to impact the landfill life and to improve the analyzed indicators.

Keywords: Integral waste management, mathematical model, dynamic model.

Resumen gráfico: representación de algunos de los resultados más importantes obtenidos a partir de la investigación y el modelo obtenido. Según su eficiencia, los escenarios se ordenan así: cuarto, tercero, primero y, por último, el segundo.

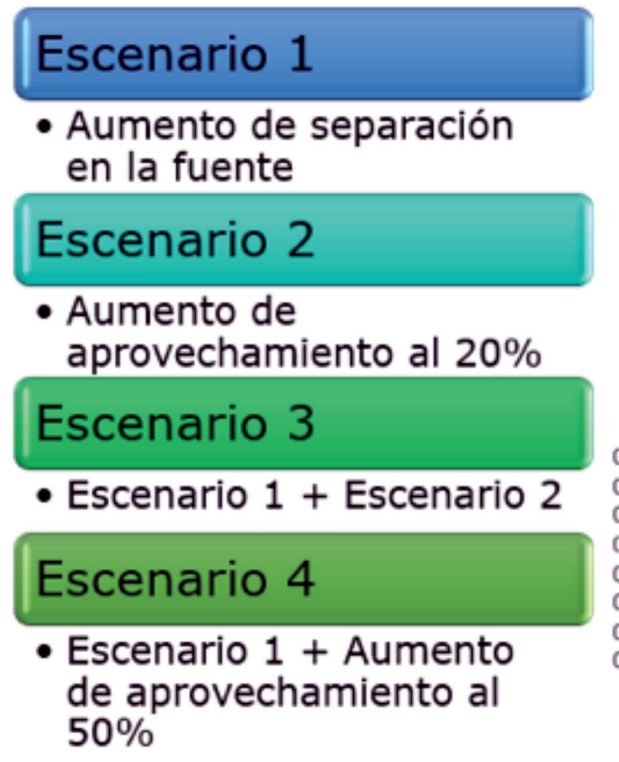

\section{INTRODUCCIÓN}

El aumento desmesurado de la población ha generado un incremento de emisiones atmosféricas, vertimientos con cargas contaminantes, aumento de generación y disposición de residuos sólidos, ampliación del espacio ocupado por las personas, entre otros factores que afectan la estabilidad social y la calidad

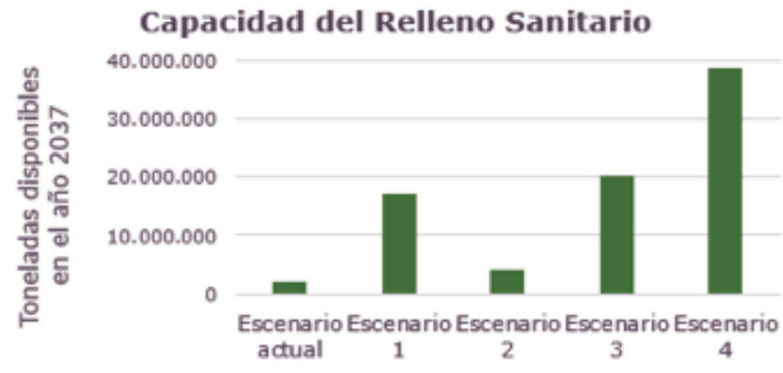

Indicador Residuos Aprovechados

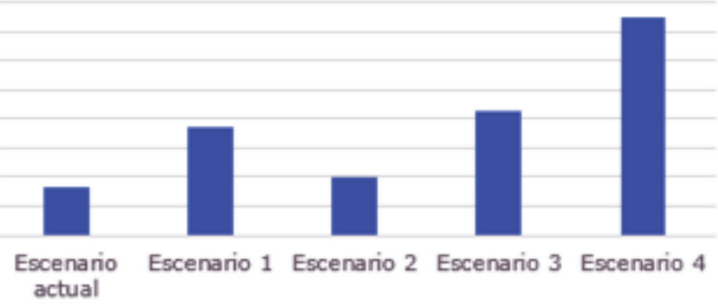

ambiental en zonas rurales y urbanas. Colombia cuenta con alrededor de 48 millones de habitantes, de los cuales más del $70 \%$ vive en zonas urbanas (Departamento Administrativo Nacional de Estadística, 2015). Lo anterior ha traído como desafío el incremento de abastecimiento de servicios públicos y saneamiento 
básico, de lo cual, tal como en la mayoría de países en desarrollo, uno de los aspectos más críticos es el manejo de residuos sólidos (Sukholthaman y Sharp, 2016).

Para hacer referencia a un caso más específico, se evidencia la problemática ambiental en Bogotá, ciudad que ha crecido muy rápido en infraestructura de servicios y vivienda, debido al mismo incremento de la población. Por ejemplo, para el 2005, Bogotá tenía 6.840.116 habitantes (DANE, 2005) y se espera que para el 2020, tenga una población de 8.380.801 personas, lo que representa un incremento del 22,5 \% en 15 años - o de 1,5\% anual - (DANE, 2009). Este importante crecimiento demográfico ha traído consigo más generación de residuos sólidos, aspecto que ha tenido una tendencia creciente desde 1998, pues desde ese año hasta el 2013, este aspecto aumentó, en promedio, 2,25\% anualmente. Lo anterior, sumado a aspectos como la informalidad de los recicladores, la poca eficiencia de algunas rutas de recolección, la falta de infraestructura que permita hacer tratamiento adecuado a los residuos, la nula o poca separación en la fuente y demás situaciones, ha sido causa del crítico escenario actual (UAESP, 2014).

El actual Plan de Gestión Integral de Residuos Sólidos (PGIRS) de Bogotá tiene como uno de sus principales objetivos establecer medidas que incluyan a los recicladores de oficio, para que se pueda crear un nuevo esquema de gestión más efectivo. En una revisión de dicho documento, se encontró que los materiales que más se disponen en el relleno sanitario son materia orgánica [42,24\%], plástico $[23,39 \%]$, papel $[9,41 \%]$, textiles $[8,13$ \%] y cartón [6,26 \%] (Secretaría del Hábitat y UAESP, 2016). Lo anterior permite ver que la disposición final y todo el proceso detrás de ella están fallando completamente, pues si se hace una comparación con algunos países desarrollados, estos suelen tener un porcentaje menor de materia orgánica. Por ejemplo, en Alemania se recolecta para llevar a disposición $14 \%$ de este tipo de residuo, en Estados Unidos el valor es de $25 \%$ y en Japón es de $26 \%$ (Sukholthaman y Sharp, 2016).

La gestión de residuos es un servicio público que, como cualquier otro, debe prestarse efectiva y equitativamente para garantizar el bienestar de la población (Sukholthaman y Sharp, 2016); sin embargo, actualmente, en Bogotá, y en Colombia, no se presta adecuadamente, por lo cual el Gobierno se planteó la idea de medir la eficiencia de este y otros procesos. Para lograr lo anterior, se tomó como base la calidad ambiental urbana, la cual resulta de las múltiples relaciones entre diferentes aspectos humanos y ambientales que llevan a afectar positiva o negativamente la calidad de vida de la población; la disposición final de residuos, el aprovechamiento y el reciclaje son factores que están involucrados en la mencionada calidad ambiental urbana (Ministerio de Ambiente y Desarrollo Sostenible, s.f.).

De acuerdo con lo anterior, el Ministerio de Ambiente y Desarrollo Sostenible creó el Índice de Calidad Ambiental Urbana (ICAU), el cual reúne variables relevantes para medir el estado de la calidad ambiental de los municipios colombianos. Para empezar, éste índice está compuesto por diferentes indicadores, clasificados como directos (aquellos que tienen relación estrecha con aspectos ambientales) e indirectos (aquellos relacionados con aspectos sociales). Por un lado, en cuanto a los indicadores directos, se encuentran, entre otros, la superficie de área verde por habitante, la calidad del aire, la calidad del agua superficial, el porcentaje de residuos sólidos aprovechados y el porcentaje de superficie construida con criterios de sostenibilidad. Por otro lado, entre los indirectos están el consumo residencial 
de agua por habitante, el consumo residencial de energía por habitante, el porcentaje de residuos sólidos dispuestos adecuadamente, espacio público efectivo por habitante y otros (Díaz, Granados y Valdés, 2016).

Debido a la problemática latente, en este artículo se presenta un modelo desarrollado con la metodología de Dinámica de Sistemas, con el cual se logró representar sistémicamente la gestión de residuos de Bogotá. Teniendo en cuenta que dicha situación se basa en aspectos como la cantidad de habitantes, las pocas estrategias de reutilización y reciclaje, y la crisis que desde ya se está sufriendo por el fin de la vida útil del Relleno Sanitario Doña Juana, se simularon distintos escenarios de gestión, incluidos los indicadores de porcentaje de residuos sólidos aprovechados y dispuestos adecuadamente como los únicos parámetros del ICAU por evaluar en este trabajo.

Como se puede notar, estos dos indicadores son importantes para determinar la calidad ambiental de la ciudad, sin embargo, en el Índice de Calidad Ambiental Urbana manejado en el país no se encuentran datos de residuos sólidos aprovechados para Bogotá y el indicador de residuos dispuestos adecuadamente de la ciudad arroja un resultado calificado como "bajo" (Díaz, Granados y Saldaña, 2015). Se evidencia, entonces, la necesidad de plantear estrategias y actividades para evaluar escenarios donde se incremente el aprovechamiento de residuos y así se disminuya la cantidad que se destina a disposición final, por lo cual el objetivo de esta investigación es elaborar un modelo para la evaluación dinámica de la gestión de residuos ordinarios en Bogotá y su influencia en el ICAU.

\section{MATERIALES Y MÉTODOS}

La metodología consistió principalmente en 3 etapas principales: revisión bibliográfica internacional, contextualización de información para el modelamiento del problema en Bogotá y modelamiento con Dinámica de Sistemas.

Revisión bibliográfica: En la revisión del estado del arte internacional, se recopiló información y resultados de investigaciones similares a la de esta propuesta, las cuales vinculaban el modelamiento para la gestión de residuos. Se encontró un modelo de gestión de residuos para Bangkok, Tailandia, donde se propusieron distintos escenarios de separación en la fuente, con los que, después de cuatro años, se lograron cambios importantes en la vida útil del Relleno Sanitario (Sukholthaman y Sharp, 2016).

De igual manera, Zanjani, Kiani, Saeedi y Vosoogh (2012) plantearon un modelo para la gestión de residuos de Teherán, Irán, incluyendo variables económicas y políticas en el mismo. Esto les permitió llegar a conclusiones más específicas, como aquella que afirma que lo más importante, en lugar de introducir el concepto de separación en la fuente, es la planeación adecuada, la aplicación de políticas públicas, la participación constante y efectiva de la sociedad y el acceso a información actualizada, las cuales son las verdaderas herramientas para que la gestión de residuos sea eficiente ambiental, social y económicamente.

Similar al caso anterior, Sufian y Bala (2007), modelaron la gestión de residuos sólidos de Daca, Bangladesh, cuya conclusión es que la generación de energía a partir de los residuos es una buena estrategia para mitigar impactos ambientales. Se encontraron investigaciones más específicas, como la de Hénault-Ethier, Martin y Housset (2017), en la que desarroIlaron un modelo para la gestión de residuos orgánicos en Quebec, Canadá y Ding, Yi, Tam, y Huangl (2016), lo hicieron para residuos de construcción en China. 
Contextualización de información para el modelamiento del problema: para tener información más cercana al caso de Bogotá, se indagaron otros artículos, tales como el de Sáez y Urdaneta (2014), quienes realizaron una revisión bibliográfica sobre la gestión de residuos sólidos en algunos países de América Latina y el Caribe. Porras (2018), relacionó el incremento poblacional del municipio de Chía con la creciente generación de residuos sólidos y creó planes de acción para mejorar todo el sistema de gestión.

Ávila, Nieto, Jiménez y Osorio (2011), a través del software Vensim, propusieron un modelo de gestión de residuos en Cali y analizaron distintas estrategias para prolongar la vida útil del relleno sanitario.

Finalmente, Sánchez (2012), realizó una investigación sobre las posibles consecuencias de implementar la estrategia complementaria de incinerar una mayor cantidad de residuos en Bogotá y prolongar la vida útil del relleno Doña Juana.

Además de investigaciones similares, se recopilaron datos cuantitativos para la modelación, por lo cual se consultó el PGIRS (2016), obtenido mediante un derecho de petición realizado a la Unidad Administrativa Especial de Servicios Públicos (UAESP). El documento proporcionó información acerca de la cantidad de residuos dispuestos en el Relleno Sanitario y la vida útil del mismo, aprovechamiento, frecuencias de recolección, residuos recolectados y caracterización de los mismos, papel de los recicladores, los prestadores y los operadores del servicio público de aseo, proyecciones (de población, de generación de residuos y económicas), problemas de la gestión actual, objetivos y metas, entre otros aspectos que fueron importantes para esta propuesta.

También se consultaron fuentes como el DANE para revisar datos demográficos; el
Ministerio de Ambiente y Desarrollo Sostenible, como base para la implementación de los indicadores del Índice de Calidad Ambiental Urbana; la UAESP y la Secretaría Distrital de Ambiente también fueron consultadas para comparar los datos obtenidos del PGIRS y recopilar otros que no estuvieran disponibles en dicho documento.

Lo más importante de la revisión bibliográfica fue hacer un análisis de contenido, mediante el cual se interpretó, comparó, ordenó y priorizó (por año e importancia) la información obtenida, para generar resultados muy cercanos a la realidad.

\section{Modelamiento con Dinámica de Sistemas:} teniendo en cuenta la información y los datos reunidos, se definieron las variables más importantes del estudio: población, recolección, disposición final, aprovechamiento de residuos orgánicos y reciclaje, debido a que son comunes en los modelos estudiados e importantes en la gestión de residuos sólidos de la ciudad. El análisis de los anteriores aspectos fue histórico, es decir, se revisó su comportamiento en los últimos años, para establecer una tendencia pasada y poder predecir tendencias futuras, las cuales se comprobarán con el modelo.

La Dinámica de Sistemas es una metodología creada por Forrester en el MIT y puede entenderse como una herramienta que muestra cómo ciertos objetos, procesos o sistemas cambian a través del tiempo (Forrester, 1999). El concepto de sistema es importante para esta metodología y se entiende como una unidad formada por diferentes elementos que interactúan de manera coordinada para cumplir un determinado fin. La dinámica de sistemas también ayuda a entender cómo se relacionan las variables y permite mostrar las posibles consecuencias de la alteración de una sola de ellas (Aracil, 1995; Sukholthaman y Sharp, 2016). 
Para la Dinámica de Sistemas, es importante la creación de estructuras de realimentación (Zanjani, Kiani, Saeedi, y Vosoogh, 2012), las cuales se definen como representaciones formales de los factores más importantes de un problema y sus respectivas relaciones (Ibarra y Redondo, 2015).

Luego de la conceptualización e identificación del problema, la Dinámica de Sistemas busca la formulación y la evaluación del mismo. La formulación, por su parte, se define como la creación de un diagrama de niveles y flujos, a través de un software específico. La evaluación es el paso en el que se demuestra el comportamiento de las variables, se ajustan a la realidad y se modelan diferentes escenarios (Redondo et al, 2018).

\section{RESULTADOS}

\section{Dinámica de Sistemas:}

a. Diagrama causal: de acuerdo con la metodología, lo primero que se obtuvo fue el diagrama causal (Figura 1) y se puede entender a partir de la población. Factores como los nacimientos, las muertes, la emigración y la inmigración afectan la cantidad de personas de la ciudad de Bogotá; sin embargo, para sintetizar estas relaciones, se usa el concepto de incremento neto, que reúne las variables mencionadas anteriormente y que llevan, en conjunto, a tener un efecto en la población. Por lo tanto, si hay un incremento neto positivo, la población aumentará y si esta última aumenta, el incremento neto también lo hará, por lo cual dicho bucle es de refuerzo.

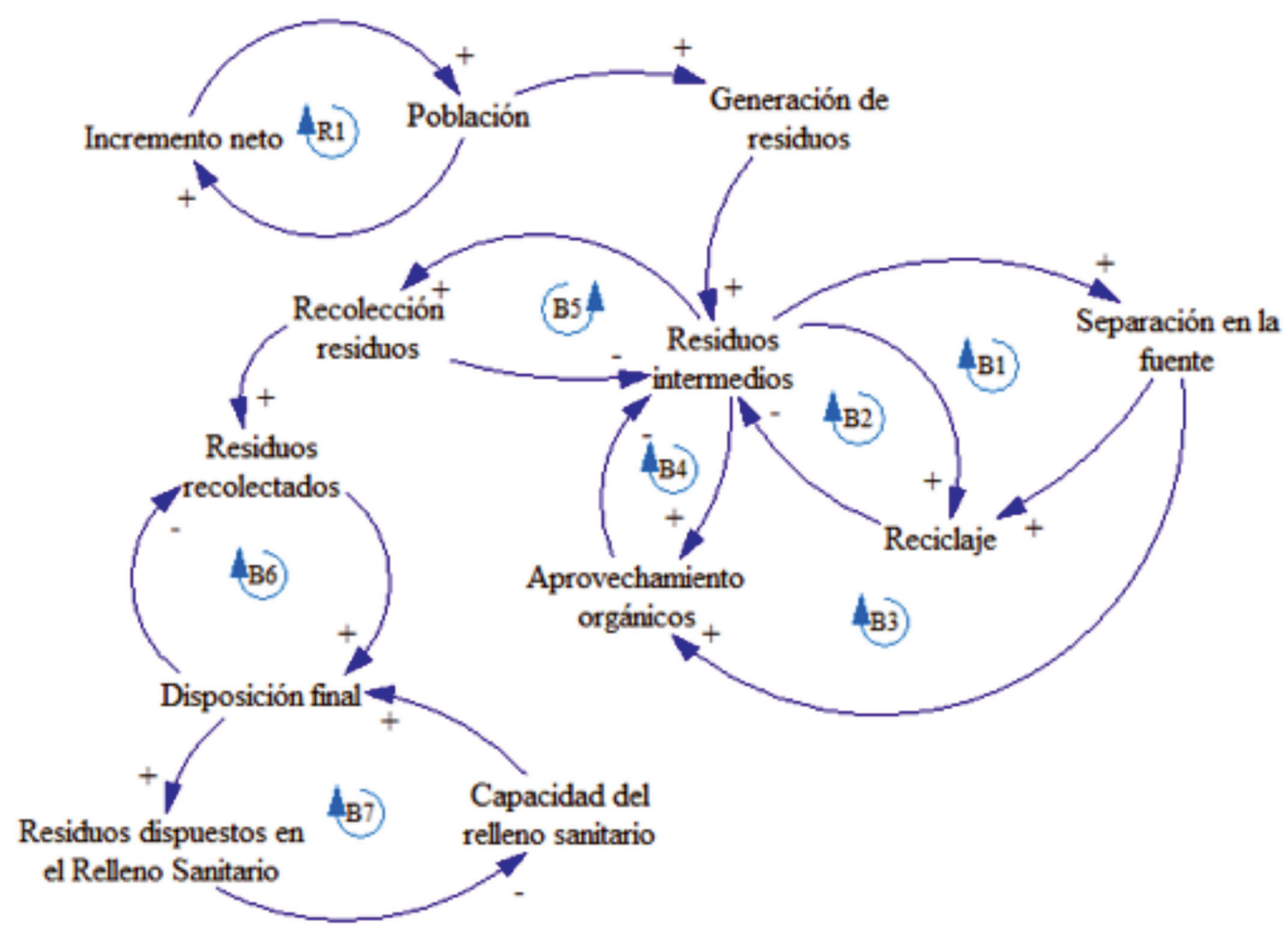

Figura 1. Diagrama causal de la gestión de residuos en Bogotá. B: Bucles de Balance; R: Bucle de Refuerzo. Fuente: Autores. 
Si la población incrementa, habrá más generación de residuos, por lo tanto, habrá más residuos intermedios, definidos en el modelo como aquellos que resultan después de los procesos de aprovechamiento, para ser recogidos y destinados a disposición en relleno sanitario. Lo que ocurre, entonces, es que si se aplican diferentes estrategias, dichos residuos pueden disminuir; por ejemplo, entre más residuos intermedios, se hará más separación en la fuente, lo que permitirá dos acciones: la primera, más reciclaje $y$, por lo tanto, menos residuos intermedios (primer bucle de balance, del que se genera otro entre los residuos y el reciclaje); la segunda, más aprovechamiento de material orgánico y menos residuos intermedios (tercer bucle de balance, del que se genera otro entre los residuos y el aprovechamiento).

Los residuos intermedios también disminuyen con la recolección, puesto que pasan a ser residuos recolectados, aquellos que van en el vehículo transportador. De esta forma, se crea el quinto bucle de balance, pues entre más residuos acumulados, habrá más recolección, pero si hay más recolección, los residuos acumulados disminuirán.
Con la recolección, aumentan los residuos recolectados, los cuales deben disponerse, es así como a mayor cantidad de residuos recolectados, más disposición final debe hacerse, pero a mayor disposición final, aquellos residuos presentes en los vehículos disminuirán; lo anterior permitió crear el sexto bucle de balance.

Finalmente, si se hace más disposición final, habrá mayor cantidad de residuos en el ReIleno Sanitario, lo que hará que la capacidad del mismo disminuya; al observar este último aspecto detalladamente, se puede ver que, a menor capacidad del relleno, se podrá hacer menos disposición final de residuos, lo que permite crear el último bucle de balance.

Diagrama de niveles y flujos: luego del diagrama causal, la traducción a diagrama de niveles y flujos se realizó con la ayuda del software Vensim Ple y el resultado se observa en la figura 2; para esto, fue importante definir adecuadamente las variables de nivel y auxiliares, así como los flujos de entrada y salida. 


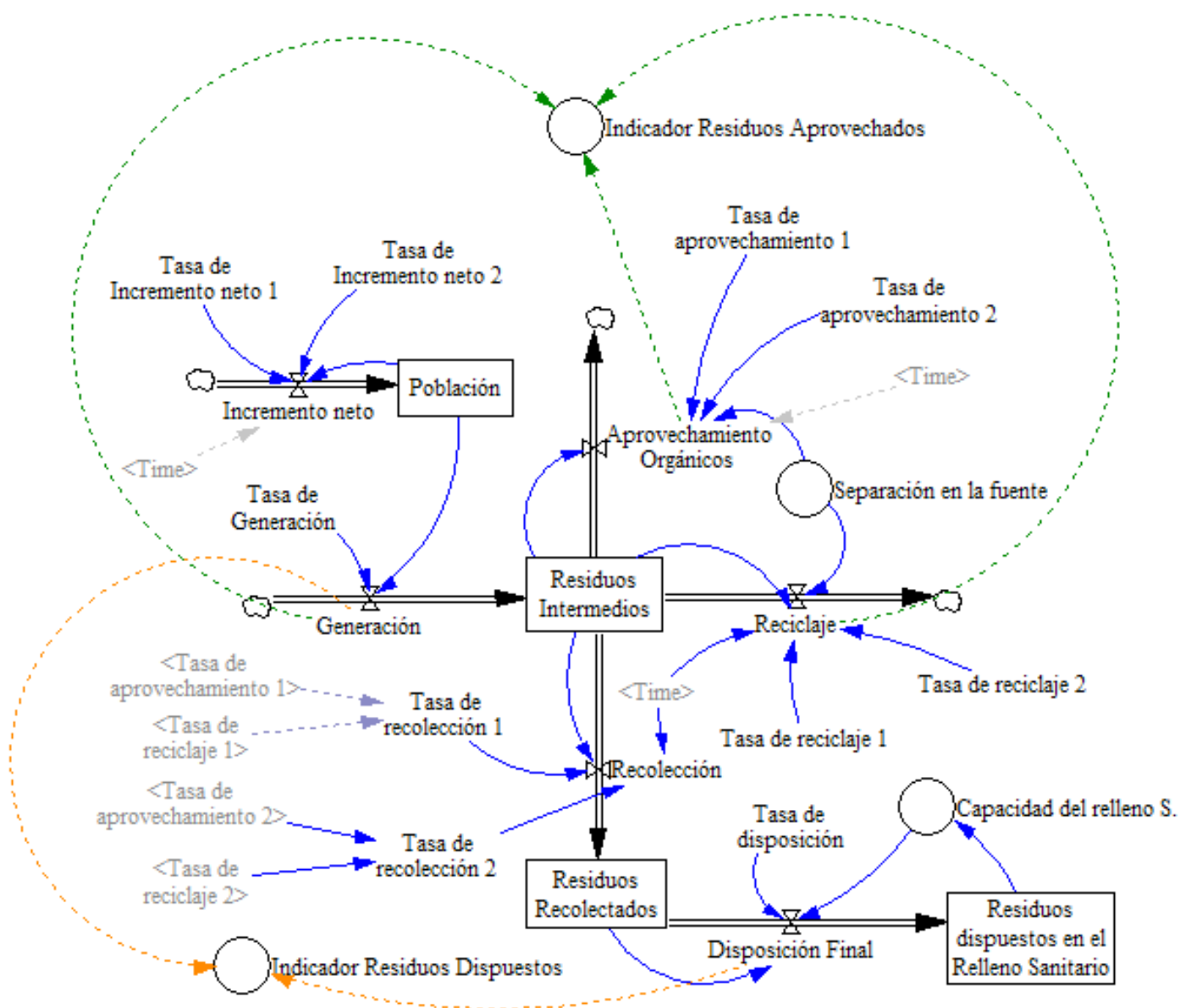

Figura 2. Diagrama de niveles y flujos de la gestión de residuos en Bogotá.

Fuente: Autores.

$$
\begin{array}{ll}
\text { \%Residuos sólidos aprovechados }=\frac{\text { Residuos Sólidos Aprovechados }}{\text { Residuos Sólidos generados }} * 100 & (\text { Ecuación 1) } \\
\text { \%Residuos dispuestos adecuadamente }=\frac{\text { R.S.Dispuestos adecuadamente }}{\text { Residuos Sólidos generados }} * 100 & \text { (Ecuación 2) }
\end{array}
$$

Para iniciar, en este diagrama se realizó la vinculación de los indicadores seleccionados del ICAU. Para desarrollar este paso adecuadamente, las ecuaciones 1 y 2 se tomaron como referencia; como se puede notar, en ellas se involucran las variables de residuos sólidos aprovechados, residuos sólidos generados y residuos sólidos dispuestos adecuadamente, las cuales se encuentran implícitas en el modelo.
Para terminar con la creación del diagrama de niveles y flujos, se elaboró el modelo matemático, a través de ecuaciones de nivel, de flujo y auxiliares. A continuación, se realizó el análisis dimensional, herramienta que verifica la coherencia de las dimensiones (o unidades) en las dos partes de una ecuación, para determinar si ésta es realmente aplicable (dimensionalmente homogénea) (Martínez, 2004). 
De acuerdo con esto, en la tabla 1 se pueden ver todas las variables incluidas en el modelo y cómo estas se simbolizan en el uso de las ecuaciones.

Tabla 1. Denominación y representación de las variables.

\begin{tabular}{l|c} 
Variable & Representación \\
\hline Población & $\mathrm{P}$ \\
\hline Residuos Intermedios & $\mathrm{RI}$ \\
\hline Residuos Recolectados & $\mathrm{RR}$ \\
\hline Residuos Dispuestos en el Relleno Sanitario & $\mathrm{IN}$ \\
\hline Incremento neto & $\mathrm{G}$ \\
\hline Generación & $\mathrm{A}$ \\
\hline Aprovechamiento de orgánicos & $\mathrm{Recicl}$. \\
\hline Reciclaje & $\mathrm{Recol}$ \\
\hline Recolección & $\mathrm{DF}$ \\
\hline Disposición Final & $\mathrm{Ti}$ \\
\hline Tasa de incremento neto & $\mathrm{Tg}$ \\
\hline Tasa de generación & $\mathrm{Trcn}$ \\
\hline Tasa de recolección & $\mathrm{Ta}$ \\
\hline Tasa de aprovechamiento & $\mathrm{Tr}$ \\
\hline Tasa de reciclaje & $\mathrm{Td}$ \\
\hline Tasa de disposición & $\mathrm{SF}$ \\
\hline Separación en la fuente & $\mathrm{CR}$ \\
\hline Capacidad del relleno sanitario & $\mathrm{I}_{1}$ \\
\hline Indicador residuos aprovechados & $\mathrm{I}_{2}$ \\
\hline Capacidad total del relleno sanitario & $\mathrm{CTR}$ \\
\hline & \\
\hline
\end{tabular}

De acuerdo con lo anterior, a continuación, se establecen las ecuaciones usadas en el modelo y su respectivo análisis dimensional:

\section{Ecuaciones de nivel:}

Población: $\frac{d P}{d t}=I N$ (Ecuación 3) $[h a b / a \tilde{n} o]=[h a b / a \tilde{n} o]$
Residuos intermedios:

$$
\begin{gathered}
\left.\frac{d R I}{d t}=G-A-\text { Recol. }- \text { Recicl. } \text { (Ecuación } 4\right) \\
{[\text { Ton/año }]=[\text { Ton/año }]-[\text { Ton/año }]-[\text { Ton/año }]-[\text { Ton/año }]} \\
{[\text { Ton/año }]=[\text { Ton/año }]}
\end{gathered}
$$


Residuos recolectados:

$$
\frac{d R R}{d t}=\text { Recol. }-D F \text { (Ecuación 5) }
$$

$[$ Ton/año $]=[$ Ton/año $]-[$ Ton $/ a \tilde{n} o]$

$[$ Ton $/ a \tilde{n} o]=[$ Ton $/ a \tilde{n} o]$

Residuos dispuestos en el Relleno Sanitario:

$$
\begin{aligned}
& \frac{d R D}{d t}=D F(\text { Ecuación 6) } \\
& {[\text { Ton/año }]=[\text { Ton/año }]}
\end{aligned}
$$

\section{Ecuaciones de flujo:}

Incremento neto:

$$
\begin{gathered}
I N=P * t i(\text { Ecuación } 7) \\
{[h a b / a \tilde{n} o]=[h a b] *[\% / a \tilde{n} o]} \\
{[h a b / a \tilde{n} o]=[h a b / a \tilde{n} o]}
\end{gathered}
$$

Generación:

$$
\begin{gathered}
G=P * \operatorname{tg}(\text { Ecuación } 8) \\
{[\text { Ton } / a \tilde{n} o]=[\text { hab }] *[\text { Ton } /(\text { hab } * a \tilde{n} o)]} \\
{[\text { Ton } / \text { año }]=[\text { Ton } / \text { año }]}
\end{gathered}
$$

Aprovechamiento:

$$
\begin{gathered}
A=R I * S F * \text { ta }(\text { Ecuación 9) } \\
{[\text { Ton } / \text { año }]=[\text { Ton }] *[1] *[\% / \text { año }]} \\
{[\text { Ton } / \text { año }]=[\text { Ton } / \text { año }]}
\end{gathered}
$$

Reciclaje:

Recicl. $=R I * S F * \operatorname{tr}$ (Ecuación 10)

$[$ Ton $/$ año $]=[$ Ton $] *[1] *[\% / a \tilde{n} o]$

$[$ Ton $/ a \tilde{n} o]=[$ Ton $/ a \tilde{n} o]$

Recolección:

$$
\begin{gathered}
\text { Recol. }=R I * \operatorname{trcn}(\text { Ecuación 11) } \\
{[\text { Ton } / \text { año }]=[\text { Ton }] *[\% / \text { año }]} \\
{[\text { Ton } / \text { año }]=[\text { Ton } / \text { año }]}
\end{gathered}
$$

Disposición Final:

$$
\begin{gathered}
D F=R R * t d(\text { Ecuación 12) } \\
{[\text { Ton/año }]=[\text { Ton }] *[\% / \text { año }]} \\
{[\text { Ton } / \text { año }]=[\text { Ton } / \text { año }]}
\end{gathered}
$$

\section{Ecuaciones auxiliares:}

Capacidad del Relleno Sanitario:

$$
\begin{gathered}
C R=C T R-R D(\text { Ecuación 13) } \\
{[\text { Ton }]=[\text { Ton }]-[\text { Ton }]} \\
{[\text { Ton }]=[\text { Ton }]}
\end{gathered}
$$

Indicador Residuos Aprovechados:

$$
\begin{gathered}
I_{1}=\frac{A+\text { Recicl. }}{G}(\text { Ecuación } 14) \\
I_{1}=\frac{[\text { Ton } / \text { año }]+[\text { Ton } / \text { año }]}{[\text { Ton } / \text { año }]} \\
I_{1}=[1]
\end{gathered}
$$

Indicador Residuos Dispuestos:

$$
\begin{gathered}
I_{2}=\frac{D F}{G}(\text { Ecuación 15) } \\
I_{2}=\frac{[\text { Ton } / \text { año }]}{[\text { Ton } / \text { año }]} \\
I_{2}=[1]
\end{gathered}
$$

2. Simulación del modelo: luego de estructurar el problema de forma cualitativa, se vinculó la información cuantitativa, mediante la simulación del modelo con los datos obtenidos en la revisión bibliográfica. El software Vensim Ple se utilizó para realizar las respectivas simulaciones, entendidas como experimentaciones con el sistema (Aracil, 1995) y se obtuvieron los primeros resultados por medio del diagrama de niveles y flujos y el modelo matemático.

En la tabla 2 se muestran los datos usados como condiciones iniciales de cada variable, así como su respectiva obtención. 
Tabla 2. Parámetros y Condiciones iniciales del Modelo.

\begin{tabular}{|c|c|c|c|c|}
\hline Variable & Valor & Unidades & Año & Fuente \\
\hline Población (P) & 6.824 .507 & Habitantes & 2006 & DANE, 2015. \\
\hline Residuos Intermedios (RI) & 2.132 .328 & Ton & 2006 & Residuos generados. UAESP, 2014. \\
\hline Residuos Recolectados (RR) & 2.009 .079 & Ton & 2006 & $\begin{array}{l}\text { Cálculo a partir de la resta entre los } \\
\text { residuos intermedios y los aprovechados } \\
\text { (123.249 Ton/año, según la tasa de } \\
\text { aprovechamiento calculada para el 2006). }\end{array}$ \\
\hline $\begin{array}{l}\text { Residuos Dispuestos en el } \\
\text { Relleno Sanitario (RD) }\end{array}$ & 2.009 .079 & Ton & 2006 & $\begin{array}{l}\text { Suposición de cero pérdidas entre la } \\
\text { recolección y la disposición. }\end{array}$ \\
\hline Tasa de incremento neto $1\left(\mathrm{ti}_{1}\right)$ & 1,426 & \%/año & $\begin{array}{l}2006- \\
2015\end{array}$ & $\begin{array}{l}\text { Cálculo a partir de los incrementos anuales } \\
\text { de la población en dicho periodo. }\end{array}$ \\
\hline Tasa de incremento neto $2\left(\mathrm{ti}_{2}\right)$ & 1,244 & \%/año & $\begin{array}{l}2016- \\
2020\end{array}$ & $\begin{array}{l}\text { Cálculo a partir de los incrementos anuales } \\
\text { de la población en dicho periodo. }\end{array}$ \\
\hline Tasa de generación (tg) & 0,312452 & $\begin{array}{l}\text { Ton/hab- } \\
\text { año }\end{array}$ & 2006 & $\begin{array}{l}\text { Cálculo a partir de los residuos intermedios } \\
\text { y la población. }\end{array}$ \\
\hline Tasa de recolección $1\left(\operatorname{trcn}_{1}\right)$ & 86,31 & \%/año & $\begin{array}{c}2006- \\
2015\end{array}$ & $\begin{array}{l}\text { Cálculo a partir de la resta entre el } 100 \% \\
\text { y las tasas de aprovechamiento y reciclaje } \\
\text { respectivas. }\end{array}$ \\
\hline Tasa de recolección $2\left(\operatorname{trcn}_{2}\right)$ & 83,41 & \%/año & $\begin{array}{c}2006- \\
2015\end{array}$ & $\begin{array}{l}\text { Cálculo a partir de la resta entre el } 100 \% \\
\text { y las tasas de aprovechamiento y reciclaje } \\
\text { respectivas. }\end{array}$ \\
\hline $\begin{array}{l}\text { Tasa de aprovechamiento } 1 \\
\left(\mathrm{ta}_{1}\right)\end{array}$ & 5,78 & \%/año & $\begin{array}{l}2006- \\
2015\end{array}$ & \multirow[b]{2}{*}{$\begin{array}{l}\text { Cálculo. Se obtuvieron los datos de } \\
\text { aprovechamiento en la ciudad en los } \\
\text { últimos años (2014-2017). Durante el } \\
\text { año } 2014 \text { se aprovechó el } 12,75 \% \text { de los } \\
\text { residuos generados, en el } 2015 \text { el valor } \\
\text { fue de } 14,62 \% \text {, en el } 2016 \text { de } 14,03 \% \\
\text { y en el } 2017 \text { de } 19,15 \% \text { (Observatorio } \\
\text { Ambiental de Bogotá, s.f.). Se generaron } \\
\text { los promedios del } 2014 \text { y } 2015 \text { ( } 13,69 \\
\% \text { para obtener la primera tasa de } \\
\text { aprovechamiento y del } 2016 \text { y } 2017 \text { ( } 16,59 \\
\% \text { para la segunda tasa. Sin embargo, este } \\
\text { valor abarca tanto aprovechamiento como } \\
\text { reciclaje, por lo cual se tuvo en cuenta la } \\
\text { caracterización del } 2016 \text { y se observó que } \\
\text { el } 42,24 \% \text { fue materia orgánica. Si se } \\
\text { relaciona este último porcentaje con los } \\
\text { primeros, se obtiene un resultado de } 5,78 \\
\% \text { de aprovechamiento de orgánicos para el } \\
\text { primer periodo y de } 7 \text { \% para el segundo. }\end{array}$} \\
\hline $\begin{array}{l}\text { Tasa de aprovechamiento } 2 \\
\left(\operatorname{ta}_{2}\right)\end{array}$ & 7 & \%/año & $\begin{array}{l}2016- \\
2020\end{array}$ & \\
\hline Tasa de reciclaje $1\left(\operatorname{tr}_{1}\right)$ & 7,91 & \%/año & $\begin{array}{c}2006- \\
2015\end{array}$ & $\begin{array}{l}\text { Cálculo. Teniendo en cuenta el valor } \\
\text { total obtenido de } 13,69 \% \text { y el valor de } \\
\text { aprovechamiento de } 5,78 \% \text {, resulta un } 7,91 \\
\% \text { para la tasa de reciclaje. }\end{array}$ \\
\hline
\end{tabular}


Continuación tabla 2

\begin{tabular}{|c|c|c|c|c|}
\hline Variable & Valor & Unidades & Año & Fuente \\
\hline Tasa de reciclaje $2\left(\operatorname{tr}_{2}\right)$ & 9,59 & \%/año & $\begin{array}{l}2016- \\
2020\end{array}$ & $\begin{array}{l}\text { Cálculo. Teniendo en cuenta el valor } \\
\text { total obtenido de } 16,59 \% \text { y el valor de } \\
\text { aprovechamiento de } 7 \% \text {, resulta un 9,59\% } \\
\text { para la tasa de reciclaje. }\end{array}$ \\
\hline Tasa de disposición (td) & 100 & \% / año & - & $\begin{array}{l}\text { Suposición en la que se asume que } \\
\text { todos los residuos que se recogen en los } \\
\text { correspondientes vehículos y que no fueron } \\
\text { objeto de aprovechamiento o reciclaje, se } \\
\text { llevan a disposición final. }\end{array}$ \\
\hline Separación en la fuente (SF) & $1-10$ & - & - & $\begin{array}{l}\text { Factor adimensional del modelo que incide } \\
\text { en el aprovechamiento y el reciclaje. }\end{array}$ \\
\hline $\begin{array}{l}\text { Capacidad total del relleno } \\
\text { sanitario (CTR) }\end{array}$ & 71.499 .073 & Ton & $\begin{array}{l}2006- \\
2038\end{array}$ & $\begin{array}{l}\text { Cálculo a partir de los residuos dispuestos } \\
\text { del } 2006 \text { al } 2017 \text { y de la proyección de los } \\
\text { que se dispondrán del } 2018 \text { al } 2038 \text {. Se } \\
\text { supone la creación de un nuevo relleno } \\
\text { sanitario con dicha capacidad en toneladas. }\end{array}$ \\
\hline
\end{tabular}

Calibración y validación del modelo: por un lado, la calibración es la optimización de todas las variables y flujos, a través de comparaciones de los comportamientos obtenidos en el modelo y los resultantes de la revisión bibliográfica (tendencias). Por otro lado, la validación se refiere al análisis de los errores relativos y al ajuste de los mismos para que resulten pequeños y los resultados futuros sean confiables (Méndez, 2012).

Para llevar a cabo este proceso, la variable de población es la primera que se puede analizar, pues es de la cual hay más información disponible y es la base de todo el modelo. De esta forma, para definir si la variable se comporta de acuerdo con las tendencias reales, se toma como base el año 2006, en el cual, según el DANE, había un total de 6.824.507 personas en la zona urbana de Bogotá (DANE, 2015). Teniendo en cuenta, además, las tasas de incremento neto definidas en la Tabla 2, se obtienen los resultados visibles en la Figura 3.

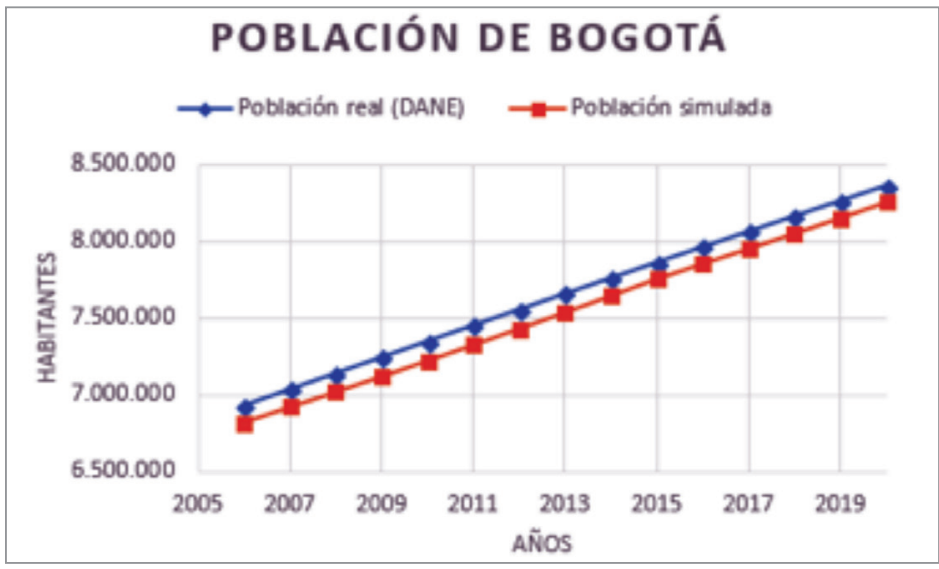

Figura 3. Comportamiento de la validación de la población. Fuente: Autores. 
De la comparación entre resultados modelados y los dados por el DANE, se puede decir que el modelo sigue la tendencia de los datos reales; además, el mayor error relativo que se obtuvo fue de $1,67486 \%$, lo que quiere decir que la diferencia entre valores no es muy grande y los datos modelados sí representan de forma cercana la realidad de la población en la ciudad.

Otra variable que se puede calibrar es la de
Residuos Dispuestos en el Relleno Sanitario, cuyo valor fue de 1.960 .000 toneladas en el 2006, 2.239.335 en el 2010 y 2.253 .072 en el 2016 (Observatorio Ambiental de Bogotá, s.f.). En este caso, tal como se observa en la figura 4, los resultados modelados reflejan una tendencia similar a los datos recolectados, aunque no sigue los comportamientos exactos; lo anterior se evidencia en los errores relativos, pues estos van desde 2,50408 \% hasta $16,59291 \%$.

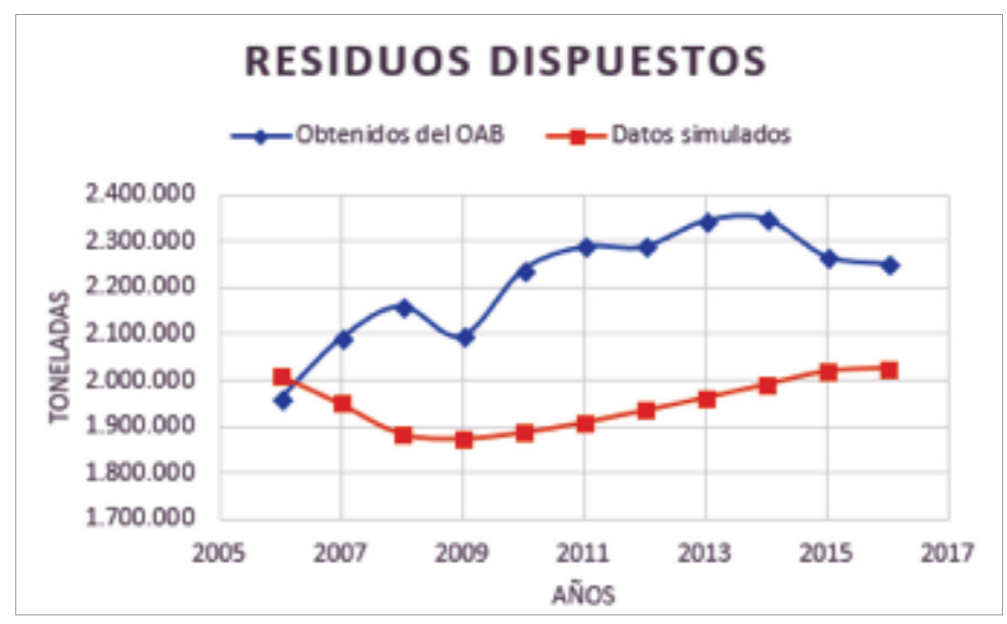

Figura 4. Comportamiento de validación de los residuos dispuestos.

Fuente: Autores.

Escenarios modelados: después de las respectivas validaciones y calibraciones, se modelan las condiciones actuales de las variables, así como también 4 simulaciones que representan diferentes estrategias que buscan mejorar la eficiencia del sistema y los resultados de los indicadores del ICAU.

El primer escenario representa un caso en el que se aumenta 3 veces la separación en la fuente y las demás condiciones se mantienen; el segundo escenario muestra un $20 \%$ de todo tipo de aprovechamiento, dividido en $8,45 \%$ para orgánicos y $11,55 \%$ de reciclaje; al tercero, con las mismas condiciones del segundo, se le suma un aumento en 3 veces la separación en la fuente; por último, el cuarto escenario se caracteriza por un $21,12 \%$ de aprovechamiento, $28,88 \%$ de reciclaje y 3 veces la separación en la fuente. 


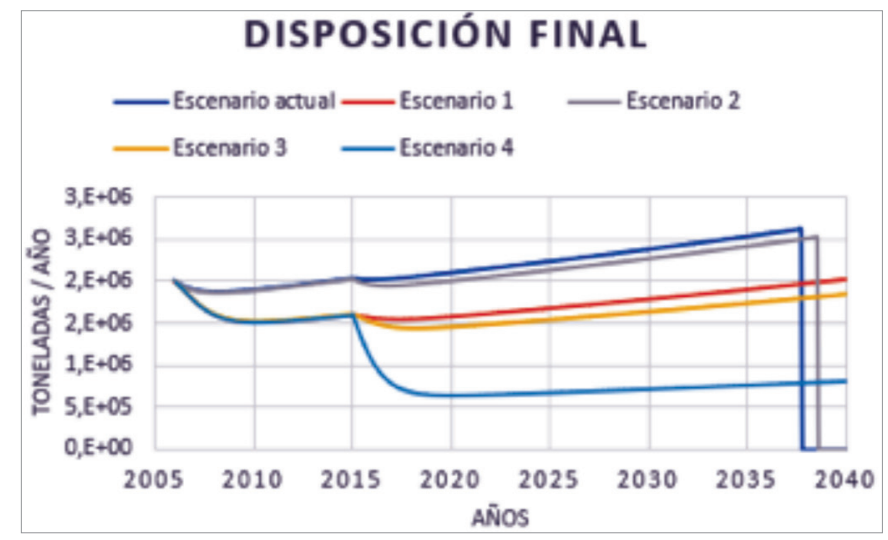

Figura 5. Comportamiento de la disposición final en diferentes escenarios.

Fuente: Autores.

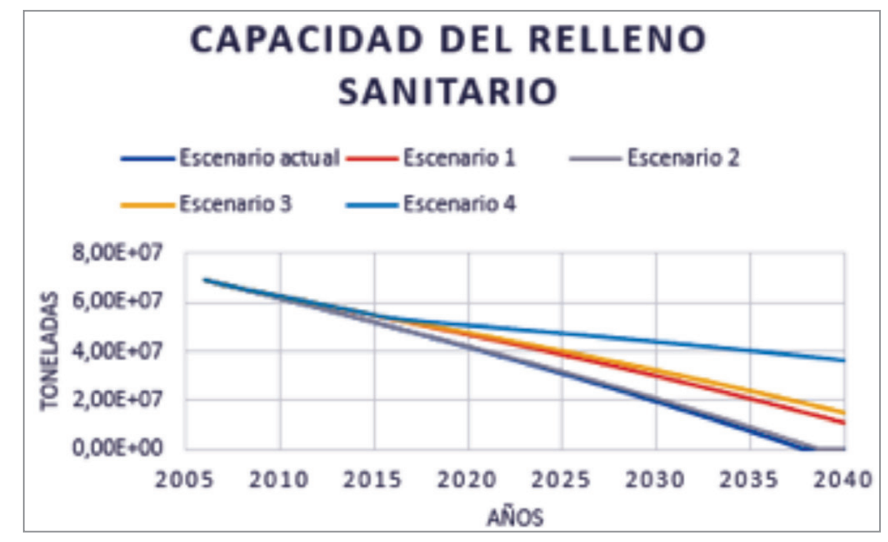

Figura 6. Comportamiento de la capacidad del relleno sanitario en diferentes escenarios.

Fuente: Autores.

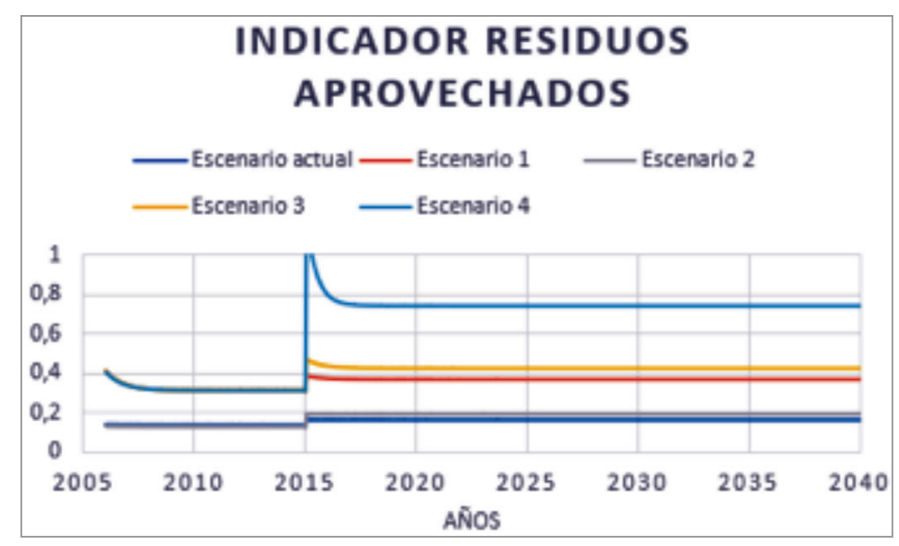

Figura 7. Comportamiento del indicador de residuos aprovechados en diferentes escenarios. Fuente: Autores. 


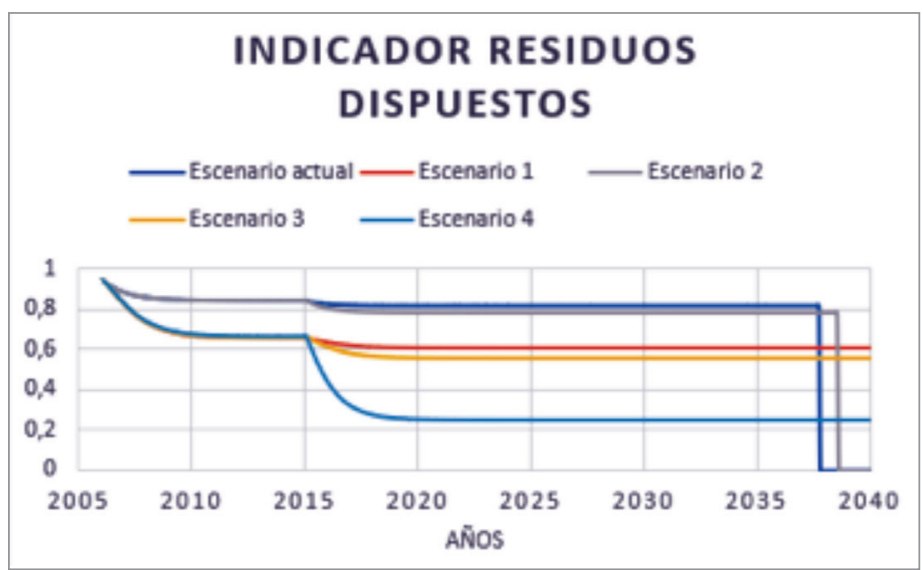

Figura 8. Comportamiento del indicador de residuos dispuestos en diferentes escenarios.

Fuente: Autores.

\section{DISCUSIÓN}

En las figuras 5 y 6 se observa claramente, que el escenario actual puede mejorar y que pueden llevarse menos residuos al relleno sanitario. El escenario 2 demuestra la menor eficiencia, debido a que en menos de un año iguala la cantidad de residuos dispuestos del escenario actual, agotando así la vida útil del relleno sanitario en un período corto. Con el escenario 1, para el 2040 se tendría espacio disponible para 11.319 .800 toneladas de residuos y con el escenario 3, para 14.934.000 toneladas.

En cuanto al indicador de residuos aprovechados, con el escenario 3 se alcanzaría un aprovechamiento constante del $42 \%$, con el escenario 1 se mantendría un valor de $37 \%$ y con el escenario 2, un aprovechamiento del $19 \%, 3$ puntos porcentuales por encima del escenario actual.

Teniendo en cuenta la ecuación 2, un valor alto del indicador de residuos dispuestos señala que más residuos del total de los generados se llevan a disposición; si el objetivo es aprovechar más residuos y disminuir de forma importante los que se llevan al relleno sanitario, es importante que este indicador tenga valores bajos. El escenario actual mantiene un valor de $81,37 \%$ de residuos dispuestos adecuadamente, el escenario 2 de 78,05 \%, el escenario 1 de $61,29 \%$ y el escenario 3 de 55,94\%.

De acuerdo con todo lo anterior, el escenario 2 , que plantea un aumento del 3,41 \% en el aprovechamiento total (tanto de orgánicos, como reciclaje), es el que genera menos mejoras, pues los resultados muestran que las tendencias se comportan de forma muy similar al escenario actual. Luego, el escenario 1, que busca aumentar únicamente la separación en la fuente, además de prolongar la vida útil del relleno sanitario, mejora en un $21 \%$ el indicador de residuos aprovechados y en 20,08 $\%$ el indicador de residuos dispuestos adecuadamente; el escenario 3, como conjunto de los dos anteriores, refleja aumento del $26 \%$ en el primer indicador $y$, comparado con el escenario actual, el segundo indicador muestra que es un $25,43 \%$ más favorable.

Finalmente, el escenario 4, que busca aumen$\operatorname{tar} 33,41 \%$ el total de aprovechamiento e incentivar la separación en la fuente, permite ver los mejores resultados, pues para el 2040 se tendría espacio disponible para 36.365 .900 
toneladas de residuos, 21.431.900 más que el escenario 3; además, el indicador de residuos aprovechados alcanza el 74,54\% y el de residuos dispuestos adecuadamente $24,54 \%$, los más favorables del análisis.

En su investigación, Sáez y Urdaneta (2014), llegaron a la conclusión de que en América Latina y el Caribe, la gestión de residuos se enfoca en la recolección y en la disposición final, sin prestar la suficiente atención a las demás variables del sistema; sumado a lo anterior, Porras (2018), afirma que, específicamente, en el departamento de Cundinamarca, los rellenos sanitarios se han tomado como la única alternativa, dejando de lado otras estrategias importantes. Bogotá no es ajena a la situación descrita y las distintas problemáticas que hoy se presentan, se han generado debido a la falta de un análisis integral del sistema.

Como se pudo ver con los distintos escenarios, la ciudad necesita plantear estrategias que tengan como objetivo incrementar el aprovechamiento de residuos y así disminuir la cantidad que se destina a disposición final.

Según el Gobierno Nacional, existen múltiples vías para mejorar el manejo de residuos sólidos de una ciudad como Bogotá, enmarcadas dentro de cuatro grandes grupos: el primero de ellos reúne estrategias para disminuir la generación de residuos, minimizar la cantidad dispuesta y promover reutilización, aprovechamiento y tratamiento de los mismos; el segundo grupo va dirigido directamente a la sociedad y hace referencia a estrategias que permitan educar a la población en este tema; el tercero está dirigido a los demás actores y busca que cada participante de la gestión asuma toda la responsabilidad y actúe para mejorar el proceso; por último, el cuarto grupo busca que las autoridades ambientales realicen controles rigurosos sobre cada actividad realizada dentro de la gestión de los residuos (CONPES 3874, 2016). Todas las estrategias antes mencionadas, son de suma importancia para el sistema, pues permiten reducir la generación de residuos, aumentar la separación y aprovechamiento de los mismos, reducir los niveles de contaminación y prolongar la vida útil del relleno sanitario (Ávila, Nieto, Jiménez y Osorio, 2011).

El análisis de los diferentes escenarios permite ver que la opción que incrementa la separación en la fuente (escenario 1) tiene un mejor resultado que aquella que plantea un mayor aprovechamiento de los residuos (escenario 2), lo que indica que es indispensable que la Alcaldía se enmarque, inicialmente, en el segundo grupo de estrategias, es decir, la educación a la población. Como lo mencionan varios autores, para obtener buenos resultados es clave sensibilizar a la población con respecto a los residuos generados en cada una de las actividades diarias e impulsar su participación responsable en el sistema de gestión (Sáez y Urdaneta, 2014; Ávila, Nieto, Jiménez y Osorio, 2011).

Desde la Alcaldía anterior se ha buscado que se seleccionen los residuos ordinarios en bolsa negra y los residuos reciclables en bolsa blanca, lo cual puede explicar el aumento del $5,12 \%$ en el aprovechamiento de residuos del 2016 al 2017. Sin embargo, se deben plantear estrategias más sólidas, que busquen una selección más estricta de los residuos, es decir que, por ejemplo, se separe materia orgánica de los residuos ordinarios y el plástico del papel y cartón; además, es claro que se debe buscar un incremento en la cantidad de personas que realicen separación en la fuente, para que toda la gestión sea más eficiente.

Establecer políticas públicas efectivas debe ser el primer paso para generar cambios positivos 
importantes en todo el sistema de gestión de residuos sólidos en Bogotá, sin embargo, para esto es fundamental el interés, la inversión y la coordinación de quienes gobiernan la ciudad, quienes tienen a cargo este servicio y todas aquellas instituciones con competencia en esta gestión (Sáez y Urdaneta, 2014; Porras, 2018); además, debe contarse con el apoyo de las autoridades ambientales, para verificar que cada actividad involucrada en la gestión de residuos se esté llevando a cabo adecuadamente y que no impacte negativamente la calidad ambiental urbana.

Continuando con la línea de estrategias, la Alcaldía, luego de promover la educación a la población, debe buscar la minimización de la cantidad de residuos que se llevan a disposición a través de incentivos a empresas o asociaciones que se dediquen a reciclar, tratar, reutilizar o aprovechar residuos. Es importante, entonces, que se eduque a la población recicladora en temas como la gestión empresarial y el Registro Único de Recicladores de Oficio (RURO); además, que se planee adecuadamente el proceso para la formalización de los mismos y que se brinden mecanismos más eficientes para la recolección selectiva. Estas estrategias atenderán el aspecto fundamental de los problemas principales del sistema de gestión de residuos en Bogotá: la informalidad, pues, tal como sucede en algunos países de América Latina y el Caribe, aún se observan personas que trabajan en las calles, separando los residuos que desde los hogares no se segregan (Sáez y Urdaneta, 2014).

La formalización de los recicladores es un punto clave, pues esto permite organizar toda la cadena desde la recolección hasta la comercialización de los nuevos productos, teniendo en cuenta aspectos como las rutas selectivas, la sectorización de la ciudad y el establecimiento formal de centros acopio, transferencia, reciclaje y aprovechamiento, tal como lo afirmó Porras (2018), como conclusión al análisis del modelo de residuos en Chía, Cundinamarca.

Además, es importante también que el Distrito tome en cuenta los registros que existen de cantidad y caracterización de los residuos sólidos que se generan en la ciudad y aquellos que Ilegan al relleno sanitario (Sáez y Urdaneta, 2014), para que a partir de todos estos datos, se planifique adecuadamente el sistema de gestión y se puedan tomar en cuenta otras estrategias alternativas al relleno sanitario, como la incineración de ciertos residuos, tal como lo registró Sánchez (2012).

Según Goicochea (2015), en los últimos años se han desarrollado distintos modelos para la gestión de residuos sólidos, vinculando variables sociales, económicas y ambientales, con el fin de apoyar la toma de decisiones; sin embargo, es importante tener en cuenta que todos los modelos tienen limitaciones, al asumir ciertas variables y no considerar todo el proceso. Por ejemplo, en Bogotá, así como en otras ciudades de América Latina y el Caribe, una de las limitaciones más grandes en la gestión de residuos sólidos es el cambio de administración, pues genera discontinuidad en todos los procesos (Sáez y Urdaneta, 2014).

\section{CONCLUSIONES}

En esta investigación, se tomó como base la creación de un nuevo relleno sanitario que pudiera funcionar hasta el 2038 con las condiciones actuales de generación, aprovechamiento y reciclaje. Se logró observar, a través de un modelo creado con ayuda del software Vensim Ple, que es urgente aumentar la separación en la fuente en los hogares bogotanos y que es importante para promover el aprovechamiento de los residuos.

Para esto, es indispensable planear y priorizar adecuadamente las estrategias que se deben impulsar, estimular la inversión en tecnologías 
para tratamiento y aprovechamiento de residuos sólidos, contar con un marco normativo sólido y acompañamiento estricto de las respectivas autoridades ambientales, iniciar un arduo plan educativo tanto para la población generadora como para la población recicladora, establecer cuántas y cuáles bolsas deben usarse para la respectiva selección de residuos, promover el desarrollo de alternativas para el manejo de residuos a través de estudios universitarios e informar a la población con datos actualizados y fácilmente entendibles.

Como se pudo ver en las diferentes simulaciones, unos escenarios generaban más beneficios que otros; sin embargo, con el más mínimo aumento de separación en la fuente o de aprovechamiento, empezaban a haber cambios importantes en toda la gestión de residuos sólidos, los cuales llevaban a prolongar la vida útil del relleno sanitario. Lo anterior quiere decir que no es urgente aumentar el aprovechamiento al $50 \%$, pero sí es importante llevar a cabo una transición lenta pero eficiente con aumentos constantes de las dos variables.

Con el aumento paulatino de la separación en la fuente y del aprovechamiento, sumado a la implementación de estrategias que mejoren las demás variables del sistema, se irá mejorando, poco a poco, el indicador de residuos aprovechados que, a su vez, contribuirá al mejoramiento del ICAU, relacionado, además, con la calidad de vida de la población de la ciudad.

De acuerdo con lo anterior, se espera que esta investigación y sus resultados sean útiles para la planeación de la gestión de residuos sólidos en Bogotá y para la aplicación de las diferentes estrategias que actualmente tiene la Alcaldía y aquellas que presente la próxima administración, a través del PGIRS, para obtener mejores resultados en los indicadores de residuos aprovechados y dispuestos.
A través de esto, se logrará que el manejo de los residuos sólidos no sea uno de los servicios públicos más críticos, sino que, por el contrario, se convierta en la vía para mejorar la calidad ambiental urbana, crear conciencia y compromiso en la población e incrementar la calidad de vida de los ciudadanos.

\section{AGRADECIMIENTOS}

Esta investigación se desarrolló gracias al Ingeniero Danny Ibarra, integrante del semillero de investigación de Ingeniería Ambiental y Bioprocesos de la Universidad Sergio Arboleda. Además, se impulsó gracias a la participación financiada por la Universidad Sergio Arboleda en el Tercer Congreso Nacional de Ciencias Ambientales, desarrollado en la ciudad de Barranquilla, del 23 al 25 de octubre de 2017.

\section{LITERATURA CITADA}

Aracil, J. (1995). Dinámica de Sistemas. Editorial Edison. Madrid, España: Editorial Edison.

Ávila, S. L., Nieto, M. S., Jiménez, D. C., y Osorio, J. C. (2011). Análisis del impacto generado en un Sistema de Gestión Integral de Residuos Sólidos por el aumento de los residuos asociados al crecimiento de la población a través de Dinámica de Sistemas. Universidad Colegio Mayor de Nuestra Señora del Rosario, Comunidad Colombiana de Dinámica de Sistemas.

CONPES 3874. Política Nacional para la Gestión Integral de Residuos Sólidos, Colombia, 21 de noviembre de 2016.

DANE, Departamento Administrativo Nacional de Estadística. (2005). Censo General 2005. Nivel Nacional.

DANE, Departamento Administrativo Nacional de Estadística. (2009). Proyecciones nacionales y departamentales de población 2005 - 2020.

DANE, Departamento Administrativo Nacional de Estadística. (2015). Estimaciones de población 1985 - 2005 y proyecciones de población 2005 2020. Total departamental por área.

Díaz, A., Granados, S., y Saldaña, A. (2015). Informe Nacional de Calidad Ambiental Urbana. Áreas urbanas con población superior a 500.000 habitantes: Colombia. Ministerio de Ambiente y Desarrollo Sostenible. 
Díaz, A., Granados, S., y Valdés, D. (2016). Índice De Calidad Ambiental Urbana-ICAU. Política de Gestión Ambiental Urbana.

Ding, Z., Yi, G., Tam, V. W., y Huang, T. (2016). A system dynamics-based environmental performance simulation of construction waste reduction management in China. Waste Management, 51, 130-141.

Forrester, J. W. (1999). Industrial dynamics. Ed. Pegasus Communications Inc.

Goicochea, O. C. (2015). Evaluación ambiental del manejo de residuos sólidos domésticos en La Habana, Cuba. Ingeniería Industrial, 36(3), 263-274.

Hénault-Ethier, L., Martin, J.P., y Housset, J. (2017). A dynamic model for organic waste management in Quebec (D-MOWIQ) as a tool to review environmental, societal and economic perspectives of a waste management policy. Waste Management, 66(1), 196-209.

Ibarra, D. W., y Redondo, J. M. (2015). Dinámica de sistemas, una herramienta para la educación ambiental en ingeniería. Revista Luna Azul, 41, 152-164.

Martínez, J. (2004). Análisis Dimensional: Apuntes de Mecánica de Fluidos. Escuela Politécnica Superior de Ingeniería de Gijón (Ed.). Universidad de Oviedo. Gijón, España.

Méndez, M. (2012). Calibración y validación del modelo hidrológico SWMM en cuencas hidrográficas de alta pendiente en Costa Rica: Tecnología En Marcha. Costa Rica.

Ministerio de Ambiente y Desarrollo Sostenible. (Sin fecha). Índice Calidad Ambiental Urbana - ICAU.

Observatorio Ambiental de Bogotá. (Sin fecha). Porcentaje de Residuos sólidos aprovechados RSA: Información detallada del indicador.

Observatorio Ambiental de Bogotá2. (Sin fecha). Disposición de Residuos en el Relleno Sanitario Doña Juana- DRRSDJ: Información detallada del indicador.
Porras, A. P. (2018). Residuos sólidos en el municipio de Chía vs crecimiento poblacional un indicador ambiental para ejecutar planes de acción enmarcados en la política pública. Universidad Militar Nueva Granada.

Redondo, J. M., Ibarra, D., Monroy, L., y Bermúdez, J. (2018). Evaluación de estrategias para la gestión integral de residuos de aparatos eléctricos y electrónicos. Dyna, 85(205), 319-327.

Sáez, A., y Urdaneta, J. A. (2014). Manejo de residuos sólidos en América Latina y el Caribe. Omnia, 20(3), 121-135.

Sánchez, J. L. (2012). Modelación de la incineración de residuos sólidos urbanos como alternativa complementaria al relleno sanitario Doña Juana en Bogotá. Pontifica Universidad Javeriana.

Secretaría del Hábitat y UAESP. (2016). Documento técnico de soporte del Plan de Gestión Integral de Residuos Sólidos para Bogotá D.C.

Sufian, M. A., y Bala, B. K. (2007). Modeling of urban solid waste management system: The case of Dhaka city. Waste Management, 27(7), 858-868.

Sukholthaman, P., y Sharp, A. (2016). A system dynamics model to evaluate effects of source separation of municipal solid waste management: $A$ case of Bangkok, Thailand. Waste Management, 52(1), 50-61.

UAESP, Unidad Administrativa Especial de Servicios Públicos. (2014). Documento para Discusión. Modelo de aprovechamiento propuesto. Recolección, transporte, clasificación y pesaje.

Zanjani, A. J., Kiani, B., Saeedi, M., y Vosoogh, A. (2012). The effect of the waste separation policy in municipal solid waste management using the system dynamic approach. International Journal of Environmental Health Engineering, 1(1), 5-10.
Conflicto de Intereses Los autores declaran no tener ningún conflicto de intereses

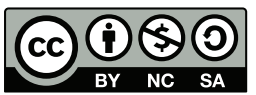

Licencia de Creative Commons

Revista de Investigación Agraria y Ambiental is licensed under a Creative Commons

Reconocimiento-NoComercial-CompartirIgual 4.0 Internacional License. 
